ELECTRONIC RESEARCH ANNOUNCEMENTS OF THE AMERICAN MATHEMATICAL SOCIETY

Volume 12, Pages 63-70 (June 16, 2006)

S $1079-6762(06) 00162-4$

\title{
ESTIMATES ON THE DIMENSION OF AN ATTRACTOR FOR A NONCLASSICAL HYPERBOLIC EQUATION
}

\author{
DELIN WU AND CHENGKUI ZHONG
}

(Communicated by Boris Hasselblatt)

\begin{abstract}
In this paper, we estimate the dimension of a global attractor for a nonclassical hyperbolic equation with a viscoelastic damping term in Hilbert spaces $H_{0}^{2} \times L^{2}$ and $D(A) \times H_{0}^{2}$, where $D(A)=\left\{v \in H_{0}^{2} \mid A v \in L^{2}\right\}$ and $A=\Delta^{2}$. We obtain an explicit formula of the upper bound of the dimension of the attractor. The obtained dimension decreases as damping grows and is uniformly bounded for large damping, which conforms to physical intuition.
\end{abstract}

\section{INTRODUCTION}

We are concerned with the estimates on the dimension of a global attractor for the following initial value problem for a nonclassical hyperbolic equation with a viscoelastic damping term:

$$
\left\{\begin{array}{l}
u_{t t}+\Delta^{2} u+\delta u_{t}+k u^{+}+g(u)=h, \quad(x, t) \in \Omega \times R^{+}, \\
u(x, 0)=u_{1}(x) \text { and } u_{t}(x, 0)=u_{2}(x), \\
u(x, t)=\nabla u(x, t)=\Delta u(x, t)=0, \quad x \in \Gamma, t \in R^{+},
\end{array}\right.
$$

where $\Omega \subset R^{2}$ is an open bounded set of $R^{2}$ with a smooth boundary $\Gamma$ sufficiently regular, and the initial data $u_{1}(x), u_{2}(x)$ are in appropriate function spaces, $k>0$ is a constant, and $\delta>0$ is a damping. The force $u^{+}=\max \{u, 0\}$ is the positive part of $u$. Further details can be found in [1] and [9.

Throughout the paper we use the notation $H=L^{2}(\Omega), V=H_{0}^{2}(\Omega)$, with the scalar product $(\cdot, \cdot)$ and the norm $|\cdot|$, respectively $((\cdot, \cdot))$ and $\|\cdot\|$, and the Sobolev spaces $H^{s}(\Omega)=W^{s, 2}(\Omega)$. We define $(u, v)=\int_{\Omega} u(x) v(x) d x,((u, v))=$ $\int_{\Omega} \Delta u(x) \Delta v(x) d x$, and $D(A)=\{v \in V \mid A v \in H\}$, where $A u=\Delta^{2} u$. We have

$$
D(A) \subset V \subset H=H^{*} \subset V^{*},
$$

where all embeddings are compact, and $H^{*}, V^{*}$ are the dual spaces of $H, V$, respectively. We can define the power $A^{s}$ for $s \in R$, which is also an operator on the space $D\left(A^{s}\right)$. We have $D\left(A^{0}\right)=H, \quad D\left(A^{1 / 2}\right)=V, D\left(A^{-1 / 2}\right)=V^{*}$, and write

$$
V_{2 s}=D\left(A^{s}\right), \quad \forall s \in R \text {. }
$$

Received by the editors May 26, 2005.

2000 Mathematics Subject Classification. Primary 35K57, 35B40, 35B41.

Key words and phrases. Dynamical system, attractor, nonclassical hyperbolic equation, Hausdorff and fractal dimensions.

Supported in part by the NSFC Grant (19971036) and Trans-Century Training Programme Foundation for the Talents by the State Education Commission.

(c)2006 American Mathematical Society Reverts to public domain 28 years from publication 
Applying norms on $D\left(A^{\frac{s}{4}}\right)$ equivalent to the $H^{s}(\Omega)$ norms and interpolation inequality, we have

$$
H_{0}^{s}(\Omega) \subset D\left(A^{\frac{s}{4}}\right) \subset H^{s}(\Omega) \subset H^{s_{1}}(\Omega) \quad \text { for } \quad s>s_{1} \geq 0 .
$$

We assume that $h \in H, g \in C^{2}(\Omega)$, and the function $g: R \rightarrow R$ satisfies the following conditions:

$$
\begin{gathered}
\liminf _{|s| \rightarrow \infty} \frac{P(s)}{s^{2}} \geq 0, \text { where } P(s)=\int_{0}^{s} g(\tau) d \tau, \\
\limsup _{|s| \rightarrow \infty} \frac{\left|g^{\prime}(s)\right|}{|s|^{\sigma}}=0, \text { for } 0 \leq \sigma<\infty,
\end{gathered}
$$

and there exists a positive constant $c_{0}$ such that

$$
\liminf _{|s| \rightarrow \infty} \frac{s g(s)-c_{0} P(s)}{s^{2}} \geq 0 .
$$

Furthermore, we need the following assumptions on $g^{\prime}$ :

(i) there exist $\beta \in(0,1)$ and $c_{1}$ such that

$$
\left|g^{\prime}\left(s_{1}\right)-g^{\prime}\left(s_{2}\right)\right| \leq c_{1}\left|s_{1}-s_{2}\right|^{\beta} ;
$$

(ii) $g^{\prime}$ is a bounded continuous mapping from $V$ into $\mathcal{L}(V, H)$ and a bounded mapping from $D(A)$ into $\mathcal{L}\left(V_{\alpha}, H\right)$ for some $\alpha \in[0,1)$.

Results on the existence of a solution and attractor in a bounded domain can be found in [10, 11, 7], and so on. In the book [11, the notion of dimension of a global attractor was considered. Later, V. V. Chepyzhov and A. A. Ilyin presented an approach that is well suited for studying the dimension of the global attractor arising in [3. The construction of the Hausdorff dimension and fractal dimension of global attractors admits the same upper bound under quite general assumptions. Further references are [2, 4, 5, 6, 8, and [12. However, there is not yet a formula for the upper bound of the dimension of a global attractor for a nonclassical hyperbolic equation. In this paper, we obtain a rather strict upper bound for the dimension a global attractor by careful estimations and give the optimal value for the parameter $\alpha$ that leads to the minimal estimate of the dimension of the attractor so that the value $\gamma=\gamma\left(k,\left\|g^{\prime}(\varphi)\right\|_{\mathcal{L}\left(V_{\alpha}, H\right)}\right)$ in the main estimates for the dimension is determined explicitly. The obtained dimension decreases as the damping coefficient $\delta$ grows and is uniformly bounded for large $\delta$, which conforms to physical intuition. Meanwhile, this gives us a relatively easy way to estimate the upper bound of the dimension of the attractor.

The main result is the following theorem.

Theorem 1.1. Let the nonclassical hyperbolic equation be given by equations (1.1). Assume that $g(u)$ satisfies conditions $(1.5)-(1.8)$. Then for any $\delta \geq \delta_{0}>0$, the Hausdorff and fractal dimensions of the global attractor $\mathcal{A}$ for the system (1.1) satisfy

$$
\begin{aligned}
\operatorname{dim}(\mathcal{A}) & \leq \min \left\{m \mid \frac{1}{m} \sum_{j=1}^{m} \lambda_{j}^{\alpha-1} \leq \frac{2 \lambda_{1} \delta^{2}}{\gamma^{2} \sqrt{\delta^{2}+4 \lambda_{1}}\left(\delta+\sqrt{\delta^{2}+4 \lambda_{1}}\right)}\right\} \\
& \leq \min \left\{m \mid \frac{1}{m} \sum_{j=1}^{m} \lambda_{j}^{\alpha-1} \leq \frac{2 \lambda_{1} \delta_{0}^{2}}{\gamma^{2} \sqrt{\delta_{0}^{2}+4 \lambda_{1}}\left(\delta_{0}+\sqrt{\delta_{0}^{2}+4 \lambda_{1}}\right)}\right\}
\end{aligned}
$$


where $\left\{\lambda_{j}\right\}_{j \in N}, 0<\lambda_{1} \leq \lambda_{2} \leq \cdots$, are the eigenvalues of the operator $\Delta^{2}$ with the Dirichlet boundary condition on $\Omega, \gamma=\gamma\left(k,\left\|g^{\prime}(\varphi)\right\|_{\mathcal{L}\left(V_{\alpha}, H\right)}\right)$ is a positive constant, and $\alpha=2 \sigma / p$ with $0 \leq 2 \sigma<p<\infty, p \geq 1$.

From Theorem 1.1, we see that the upper bound of the dimension is decreasing with respect to $\delta$ and remains small when damping coefficient $\delta$ is very large because

$$
\phi(\delta)=\frac{2 \lambda_{1} \delta^{2}}{\gamma^{2} \sqrt{\delta^{2}+4 \lambda_{1}}\left(\delta+\sqrt{\delta^{2}+4 \lambda_{1}}\right)}
$$

increases as $\delta$ grows and

$$
\lim _{m \rightarrow+\infty} \frac{1}{m} \sum_{j=1}^{m} \lambda_{j}^{\alpha-1}=0, \quad \lim _{\delta \rightarrow+\infty} \phi(\delta)=\frac{\lambda_{1}}{\gamma^{2}} .
$$

\section{Preliminary Results}

We use (1.2) to give the following definition of a weak solution to system (1.1).

Definition 2.1. Let $h \in H, E_{0}=V \times H, E_{1}=D(A) \times V$, and $\left\{u_{1}, u_{2}\right\} \in E_{0}$. A weak solution (1.1) is a function $u(x, t)$ such that

$$
u \in L^{2}((0, T), V), u_{t} \in L^{2}((0, T), H), u_{t t} \in L^{2}\left((0, T), V^{*}\right) ;
$$

(ii) for all $v \in C_{0}^{\infty}([0, T] \times \Omega)$, the generalized formula holds:

$$
\frac{d}{d t}\left(u_{t}, v\right)+\delta\left(u_{t}, v\right)+(\Delta u, \Delta v)+\left(k u^{+}, v\right)+(g(u), v)=(h, v) .
$$

Now we recall some basic results in [10, 11, 7].

Lemma 2.2. Suppose that the constants $T>0, k>0$ and the initial conditions $u_{1}(x) \in V$ and $u_{2}(x) \in H$ are given. Also, $g(u)$ satisfies (1.5) - (1.7). Then for system (1.1) there exists a unique (weak) solution such that

$$
u \in C([0, T], V) \quad \text { and } \quad u_{t} \in C((0, T], H) .
$$

The next two lemmas are results on the existence of a global attractor of the dynamical system $S(t), \quad t \geq 0$.

Lemma 2.3. Let $g$ satisfy (1.5) - (1.7), $h \in H$. Then the dynamical system associated to the system (1.1) possesses a global attractor $\mathcal{A}$, which is compact, connected, and maximal among the functional invariant sets in $E_{0}$.

For the proof of the main result, we use the following result, which gives additional information about the global attractor $\mathcal{A}$.

Lemma 2.4. Assume that $g$ satisfies conditions (1.5) - (1.7) and $h \in H$. Then the global attractor $\mathcal{A}$ is included and is bounded in the space $E_{1}$.

\section{The Hausdorff and fractal Dimensions of the attractor}

We shall prove in this section that the global attractor given in Lemma 2.3 is finite dimensional. For every $t \in R$, we define the mapping

$$
S(t):\left\{u_{1}, u_{2}\right\} \rightarrow\left\{u(t), u_{t}(t)\right\}
$$

which maps $E_{0}=V \times H$ and $E_{1}=D(A) \times V$ into themselves and they enjoy the group properties. Therefore, for every $t, S(t)$ is a homeomorphism from $E_{0}$ onto $E_{0}$ 
(or from $E_{1}$ onto $E_{1}$ ) and is continuous in $E_{0}$ (or $E_{1}$ ). We define the inner product in $E_{0}$ by $(\{p, q\},\{\tilde{p}, \tilde{q}\})_{E_{0}}=(p, \tilde{p})_{V}+(q, \tilde{q})_{H}$ for $p, \tilde{p} \in V$ and $q, \tilde{q} \in H$.

There exists a complete orthonormal family in $H,\left\{\omega_{j}\right\}_{j \in N}$, consisting of the eigenvectors of $A$ :

$$
\begin{gathered}
A \omega_{j}=\lambda_{j} \omega_{j}, \quad \forall j \in N, \\
0<\lambda_{1} \leq \lambda_{2} \leq \cdots, \quad \lambda_{j} \rightarrow \infty \text { as } j \rightarrow \infty .
\end{gathered}
$$

Let $\varepsilon_{0}=\min \left(\delta / 4, \lambda_{1} / 2 \delta\right)$; for any $\varepsilon \in\left(0, \varepsilon_{0}\right)$, we consider the semigroup of operators $S_{\varepsilon}(t):=R_{\varepsilon} S(t) R_{-\varepsilon}$ defined by

$$
S_{\varepsilon}(t):\left\{u_{1}, u_{2}+\varepsilon u_{1}\right\} \rightarrow\left\{u(t), u_{t}(t)+\varepsilon u(t)\right\} .
$$

The operator $R_{\varepsilon}, \varepsilon \in R$, is an isomorphism of $E_{0}$, given by the formula $R_{\varepsilon}$ : $\{x, y\} \rightarrow\{x, y+\varepsilon x\}$, for any $x, y \in E_{0}$. If $\mathcal{A}$ is the maximal attractor defined by Lemma 2.3 for $S(t)$, then $R_{\varepsilon} \mathcal{A}$ is the maximal attractor for $S_{\varepsilon}(t)$.

We first check assumption (ii) on $g^{\prime}$ for the operators $S(t)$ to give the optimal value for the parameter $\alpha$.

Lemma 3.1. There exists $\alpha \geq 0$ such that for every $\varphi \in D(A)$, the differential $g^{\prime}(\varphi)$ belongs to $\mathcal{L}\left(V_{\alpha}, H\right)$ and, for every $R>0$,

$$
K=\sup _{\|\varphi\|_{D(A)} \leq R}\left\|g^{\prime}(\varphi)\right\|_{\mathcal{L}\left(V_{\alpha}, H\right)}<\infty .
$$

Proof. From (1.6) we have

$$
\left|g^{\prime}(\varphi)\right| \leq c_{2}\left(1+|\varphi|^{\sigma}\right) \quad \text { with } \quad 0 \leq \sigma<\infty, \quad \Omega \subset R^{2} .
$$

The case $\sigma=0$ is obvious.

For $\sigma>0$, if $\varphi \in D(A)$ with $\|\varphi\|_{D(A)} \leq R$, then by (1.4) and the Sobolev imbedding inequality

$$
\left\|g^{\prime}(\varphi)\right\|_{L^{p / \sigma}} \leq c_{3}(R) \quad \text { with } \quad 0<2 \sigma<p<\infty, \quad p \geq 1 .
$$

Let $\psi \in V_{\alpha}$ and $\phi \in H$; we apply Hölder's inequality with exponents $p / \sigma$, $2 p /(p-2 \sigma), 2$, and find

$$
\left|\int_{\Omega} g^{\prime}(\varphi) \psi \phi d x\right| \leq\left\|g^{\prime}(\varphi)\right\|_{L^{p / \sigma}}\|\psi\|_{L^{2 p /(p-2 \sigma)}}\|\phi\|_{H} .
$$

For $n=2$ due to (1.4) and the Sobolev imbedding theorem we have

$$
\|\psi\|_{L^{2 p /(p-2 \sigma)}} \leq c_{4}\|\psi\|_{\alpha} \quad \text { if } \quad \alpha=\frac{2 \sigma}{p} .
$$

Therefore,

$$
\left|\int_{\Omega} g^{\prime}(\varphi) \psi \phi d x\right| \leq c_{3}(R) c_{5}\|\psi\|_{\alpha}\|\phi\|_{H} .
$$

This shows that $g^{\prime}(\varphi) \psi$ is in the space $H\left(=H^{*}\right)$ and its norm in $H$ is bounded by $c_{3}(R) c_{5}\|\psi\|_{\alpha}$, i.e., $K \leq c_{3}(R) c_{5}$.

From Lemma 3.1 we obtain the optimal value for the parameter $\alpha=2 \sigma / p$ with $0 \leq \alpha<1$ that leads to the minimal estimate of the dimension of the attractor. 
Proposition 3.2. Assume that $g$ satisfies (1.5)-(1.7). Then the linearized problem of (1.1) around the solution $u$,

$$
V_{t t}+\Delta^{2} V+\delta V_{t}+f^{\prime}(u) V+g^{\prime}(u) V=0,
$$

where $f(u)=k u^{+}$, with initial data

$$
V(x, 0)=y \in V, \quad V_{t}(x, 0)=z \in H,
$$

has a unique (weak) solution.

Proof. Using Lemma 2.2, we are able to prove that the linearized problem (3.5) (3.6) possesses a unique solution $V(t)$ such that

$$
V(t) \in C([0, T], V) \quad \text { and } \quad V_{t}(t) \in C([0, T], H) .
$$

We can then define a linear map $L\left(t, \varphi_{0}\right): E_{0} \rightarrow E_{0}$ by setting

$$
L\left(t, \varphi_{0}\right):\{y, z\} \rightarrow\left\{V(t), V_{t}(t)\right\} .
$$

It can also be proved that $L\left(t, \varphi_{0}\right)$ is bounded and that $\{S(t)\}_{t \geq 0}$ is uniformly differentiable on $\mathcal{A}$, i.e.,

$$
\frac{\left\|S(t)\left(\varphi_{0}+\eta\right)-S(t)\left(\varphi_{0}\right)-L\left(t, \varphi_{0}\right) \eta\right\|_{E_{0}}^{2}}{\|\eta\|_{E_{0}}^{2}} \rightarrow 0 \text { as } \eta=\{y, z\} \rightarrow 0 .
$$

Setting $\theta=R_{\varepsilon} \varphi=\left\{u, u_{t}+\varepsilon u\right\}$ and choosing

$$
\varepsilon=\frac{\lambda_{1} \delta}{\delta^{2}+4 \lambda_{1}}
$$

we may rewrite system (1.1) as a first evolution equation of the form

$$
\theta_{t}=B(\theta)=-A_{\varepsilon} \theta-b(\theta)+\bar{h},
$$

where $b(\theta)=\{0, G(u)\}, \bar{h}=\{0, h\}$, and

$$
A_{\varepsilon}=\left(\begin{array}{cc}
\varepsilon I & -I \\
\Delta^{2}-\varepsilon(\delta-\varepsilon) I & (\delta-\varepsilon) I
\end{array}\right) .
$$

Here $I$ denotes the identity mapping. Also for simplicity of the presentation, we denote by $\{u, v\}^{T}$ the transposed form of $\{u, v\}$. In the above notation, the first variation equation (3.5) has the form

$$
\begin{gathered}
U_{t}=B^{\prime}(\theta) U=-A_{\varepsilon} U-b^{\prime}(\theta) U, \\
U(0)=\xi,
\end{gathered}
$$

where $U=\left\{V, V_{t}+\varepsilon V\right\}, \quad b^{\prime}(\theta)=\left\{0, G^{\prime}(u) V\right\}, \quad \xi=\{y, z\} \in E_{0}$. We consider $m$ solutions $U(t)=U_{1}(t), \ldots, U_{m}(t)$ of $(3.5)-(3.6)$ corresponding to initial data $\xi=\xi_{1}, \ldots, \xi_{m}, \xi_{k} \in E_{0}, k=1,2, \ldots, m$.

For the proof of the main result we need the following lemma, which can be found in 12 .

Lemma 3.3. For any $\varphi=(y, z) \in E_{0}$,

$$
\left(A_{\varepsilon} \varphi, \varphi\right)_{E_{0}} \geq \rho\|\varphi\|_{E_{0}}^{2}+\frac{\delta}{2}|z|^{2},
$$

where

$$
\rho=\frac{\lambda_{1} \delta}{\sqrt{\delta^{2}+4 \lambda_{1}}\left(\delta+\sqrt{\delta^{2}+4 \lambda_{1}}\right)} .
$$


Recalling that in the generalized Liouville formula

$$
\begin{aligned}
\mid U_{1}(t) & \left.\wedge \cdots \wedge U_{m}(t)\right|_{\wedge^{m} E_{0}} \\
= & \left|\xi_{1}(t) \wedge \cdots \wedge \xi_{m}(t)\right|_{\wedge^{m} E_{0}} \exp \int_{0}^{t} \operatorname{Tr}\left(B^{\prime}\left(S_{\varepsilon}(\tau) \theta_{0}\right) \circ Q_{m}(\tau)\right) d \tau,
\end{aligned}
$$

we have that the $m$-trace $\operatorname{Tr}\left(B^{\prime}\left(S_{\varepsilon}(\tau) \theta_{0}\right) \circ Q_{m}(\tau)\right)$ provides information for the evolution of the $m$-dimensional volumes, transported along $S_{\varepsilon}(t) \theta_{0}$, by the first variation equation. We denote by $Q_{m}(t)$ the orthogonal projector in $E_{0}$ onto the subspace spanned by $U_{1}(t), \ldots, U_{m}(t)$. We also denote by

$$
\Phi_{j}(t)=\left\{y_{j}, z_{j}\right\}, \quad j=1, \ldots, m,
$$

the orthonormal basis of $\operatorname{span}\left\{U_{1}(t), \ldots, U_{m}(t)\right\}=Q_{m}(t) E_{0}$. We have that

$$
\begin{aligned}
\operatorname{Tr}\left(B^{\prime}\left(S_{\varepsilon}(\tau) \theta_{0}\right) \circ Q_{m}(\tau)\right) & =\sum_{j=1}^{\infty}\left[B^{\prime}\left(S_{\varepsilon}(\tau) \theta_{0}\right) \circ Q_{m}(\tau) \Phi_{j}(\tau), \Phi_{j}(\tau)\right]_{E_{0}} \\
& =\sum_{j=1}^{m}\left(B^{\prime}(\theta(\tau)) \Phi_{j}(\tau), \Phi_{j}(\tau)\right)_{E_{0}} .
\end{aligned}
$$

Under the above notation, we prove our main result.

Proof of Theorem 1.1. From Lemma 2.4 we have that $R_{\varepsilon} \mathcal{A} \subset E_{1}$. Using this fact and conditions $(1.5)-(1.8)$, we write

$$
\begin{aligned}
\left(B^{\prime}(\theta(s)) \Phi_{j}, \Phi_{j}\right)_{E_{0}} & =\left(-A_{\varepsilon} \Phi_{j}, \Phi_{j}\right)_{E_{0}}+\left(-b^{\prime}(\theta) \Phi_{j}, \Phi_{j}\right)_{E_{0}} \\
& \leq-\rho\left\|\Phi_{j}\right\|_{E_{0}}^{2}-\frac{\delta}{2}\left|z_{j}\right|^{2}+\left(\left\{0, G^{\prime}(u) y_{j}\right\},\left\{y_{j}, z_{j}\right\}\right)_{E_{0}} .
\end{aligned}
$$

Applying Lemma 3.1, we take

$$
\gamma=\sup _{|A u| \leq R}\left|G^{\prime}(u)\right|_{\mathcal{L}\left(V_{\alpha}, H\right)}=k+K<\infty .
$$

This allows us to majorize $\left|\left(G^{\prime}(u) y_{j}, z_{j}\right)\right|$ by

$$
\left|G^{\prime}(u) y_{j}\right|\left|z_{j}\right| \leq \gamma\left\|y_{j}\right\|_{\alpha}\left|z_{j}\right| .
$$

Hence, applying Lemma 3.3 and Young's inequality to relation (3.14), we deduce

$$
\begin{aligned}
& \left(B^{\prime}(\theta(s)) \Phi_{j}, \Phi_{j}\right)_{E_{0}} \\
& \quad \leq-\rho\left\|\Phi_{j}\right\|_{E_{0}}^{2}-\frac{\delta}{2}\left|z_{j}\right|^{2}+\gamma \int_{\Omega}\left\|y_{j}\right\|_{\alpha}\left|z_{j}\right| d x \\
& \quad \leq-\rho\left\|\Phi_{j}\right\|_{E_{0}}^{2}-\frac{\delta}{2}\left|z_{j}\right|^{2}+\frac{\delta}{2}\left|z_{j}\right|^{2}+\frac{\gamma^{2}}{2 \delta}\left\|y_{j}\right\|_{\alpha}^{2} \\
& \quad \leq-\rho\left\|\Phi_{j}\right\|_{E_{0}}^{2}+\frac{\gamma^{2}}{2 \delta}\left\|y_{j}\right\|_{\alpha}^{2} .
\end{aligned}
$$

Since $\left\{\Phi_{j}\right\}$ is an orthonormal basis of $Q_{m} E_{0}$, we have from (3.15) the inequality

$$
\sum_{j=1}^{m}\left(B^{\prime}(\theta(s)) \Phi_{j}(s), \Phi_{j}(s)\right)_{E_{0}} \leq-m \rho+\frac{\gamma^{2}}{2 \delta} \sum_{j=1}^{m}\left\|y_{j}\right\|_{\alpha}^{2} .
$$


Therefore, applying the result of Lemma VI.6.3 in [1] to any orthogonal family of elements $\left\{y_{j}, z_{j}\right\}, j=1, \ldots, m$, of $E_{0}$, we obtain that

$$
\sum_{j=1}^{m}\left\|y_{j}\right\|_{\alpha}^{2} \leq \sum_{j=1}^{m} \lambda_{j}^{\alpha-1} \text { with } \alpha=2 \sigma / p, \quad 0 \leq 2 \sigma<p<\infty, \quad p \geq 1 .
$$

Thus, by (3.16) and (3.17), we have the following estimate:

$$
\operatorname{Tr}\left(B^{\prime}\left(S_{\varepsilon}(\tau) \theta_{0}\right) \circ Q_{m}(\tau)\right) \leq-m \rho+\frac{\gamma^{2}}{2 \delta} \sum_{j=1}^{m} \lambda_{j}^{\alpha-1} .
$$

We integrate (3.18) with respect to time $t$ to obtain the relation

$$
q_{m}(t)=\sup _{\varphi_{0} \in R_{\varepsilon} \mathcal{A}} \sup _{\left\|\xi_{i}\right\| \leq 1} \frac{1}{t} \int_{0}^{t} \operatorname{Tr}\left(B^{\prime}\left(S_{\varepsilon}(\tau) \theta_{0}\right) \circ Q_{m}(\tau)\right) d \tau
$$

for $\xi_{i} \in E_{0}, i=1, \ldots, m$, where $q_{m}=\lim \sup _{t \rightarrow \infty} q_{m}(t)$. Hence,

$$
q_{m} \leq-m \rho+\frac{\gamma^{2}}{2 \delta} \sum_{j=1}^{m} \lambda_{j}^{\alpha-1} .
$$

We infer from (3.19) and (2.49) in [11, Chapter V] the following bound on the uniform Lyapunov exponents $\mu_{j}, j \in N$, of $\mathcal{A}\left(\right.$ or $R_{\varepsilon} \mathcal{A}$ ):

$$
\mu_{1}+\cdots+\mu_{j} \leq-m \rho+\frac{\gamma^{2}}{2 \delta} \sum_{j=1}^{m} \lambda_{j}^{\alpha-1}
$$

From (3.2), we obtain that

$$
\frac{1}{m} \sum_{j=1}^{m} \lambda_{j}^{\alpha-1} \rightarrow 0 \text { as } m \rightarrow \infty .
$$

Hence, there exists $m(\geq 1)$ such that

$$
\frac{1}{m} \sum_{j=1}^{m} \lambda_{j}^{\alpha-1} \leq \frac{2 \delta \rho}{\gamma^{2}}
$$

Consequently, we have that $q_{m} \leq 0$.

Therefore, by Theorem V.3.3 in [1] and Theorem 2.1 in [3], the dimensions of the attractor $\mathcal{A}$ are

$$
\begin{aligned}
& \operatorname{dim}_{H}(\mathcal{A}) \leq \min \left\{m \mid \frac{1}{m} \sum_{j=1}^{m} \lambda_{j}^{\alpha-1} \leq \frac{2 \lambda_{1} \delta^{2}}{\gamma^{2} \sqrt{\delta^{2}+4 \lambda_{1}}\left(\delta+\sqrt{\delta^{2}+4 \lambda_{1}}\right)}\right\}, \\
& \operatorname{dim}_{f}(\mathcal{A}) \leq \min \left\{m \mid \frac{1}{m} \sum_{j=1}^{m} \lambda_{j}^{\alpha-1} \leq \frac{2 \lambda_{1} \delta^{2}}{\gamma^{2} \sqrt{\delta^{2}+4 \lambda_{1}}\left(\delta+\sqrt{\delta^{2}+4 \lambda_{1}}\right)}\right\} .
\end{aligned}
$$

Remark 3.4. The estimate from Theorem 1.1 for the dimension of the attractor depends on the parameter $p$, which seems to be quite arbitrary. For convenience, we assume that the value of $p$ is optimal.

From (3.2) and (3.22) we deduce that $\alpha=0$, i.e., $p=+\infty$ is the optimal value for the minimal estimate of the dimension of the attractor. 


\section{REFERENCES}

[1] N. U. Ahmed and H. Harbi, Mathematical analysis of dynamic models of suspension bridges, SIAM J. Appl. Math., 58 (1998), 853-874. MR.1616611 (99d:73050)

[2] F. Balibrea and J. Valero, On dimension of attractors of differential inclusions and reactiondiffusion equations, Discrete Contin. Dynam. Systems, 5 (1999), 515-528. MR 1696326 (2000k:37122)

[3] V. V. Chepyzhov and A. A. Ilyin, On the fractal dimension of invariant sets: applications to Navier-Stokes equations, Discrete Contin. Dynam. Systems, 10 (2004), 117-135. MR2026186 (2004j:37151)

[4] P. Constantin and C. Foias, Global Lyapunov exponents, Kaplan-Yorke formulas and the dimension of the attractors for $2 D$ Navier-Stokes equations, Comm. Pure Appl. Math., 38 (1985), 1-27. MR0768102 (87g:35186)

[5] A. Eden, A. J. Milani, and B. Nicolaenko, Finite-dimensional exponential attractors for semilinear wave equations with damping, J. Math. Anal. Appl., 169 (1992), 408-419. MR1180900 (93f:35200)

[6] J. M. Ghidaglia and R. Temam, Attractors for damped nonlinear hyperbolic equations, J. Math. Pures Appl., 66 (1987), 273-319. MR0913856 (89m:35141)

[7] N. I. Karachalios and N. M. Stavrakakis, Existence of a global attractor for semilinear dissipative wave equations on $R^{N}$, J. Differential Equations, 157 (1999), 183-205. MR1710020 (2000e:35151)

[8] N. I. Karachalios and N. M. Stavrakakis, Estimates on the dimension of a global attractor for a semilinear dissipative wave equation on $R^{N}$, Discrete Contin. Dynam. Systems, 4 (2002), 939-951. MR1920653 (2003e:35199)

[9] A. C. Lazer and P. J. McKenna, Large-amplitude periodic oscillations in suspension bridges: some new connections with nonlinear analysis, SIAM Review, 32 (1990), 537-578. MR.1084570 (92g:73059)

[10] J. C. Robinson, Infinite-dimensional dynamical systems. An introduction to dissipative parabolic PDEs and the theory of global attractors, Cambridge University Press, Cambridge, 2001. MR:1881888 (2003f:37001a)

[11] R. Temam, Infinite-dimensional dynamical systems in mechanics and physics, Springer, New York, 1997. MR1441312 (98b:58056)

[12] S. F. Zhou, On dimension of the global attractor for damped nonlinear wave equations, J. Math. Phys., 40 (1999), 1432-1438. MR1674622 (2000g:35155)

School of Mathematics and Statistics, Lanzhou University, Lanzhou, 730000, P. R. CHINA

E-mail address: wudelin03@st.lzu.edu.cn

School of Mathematics and Statistics, Lanzhou University, Lanzhou, 730000, P. R. CHINA

E-mail address: ckzhong@lzu.edu.cn 